Document downloaded from:

http://hdl.handle.net/10251/63712

This paper must be cited as:

Cortés, J.; Santonja, F.; Tarazona Tornero, AC.; Villanueva Micó, RJ.; Villanueva Oller, FJ. (2015). A probabilistic estimation and prediction technique for dynamic continuous social science models: The evolution of the attitude of the Basque Country population towards ETA as a case study. Applied Mathematics and Computation. 264:13-20. doi:10.1016/j.amc.2015.03.128.

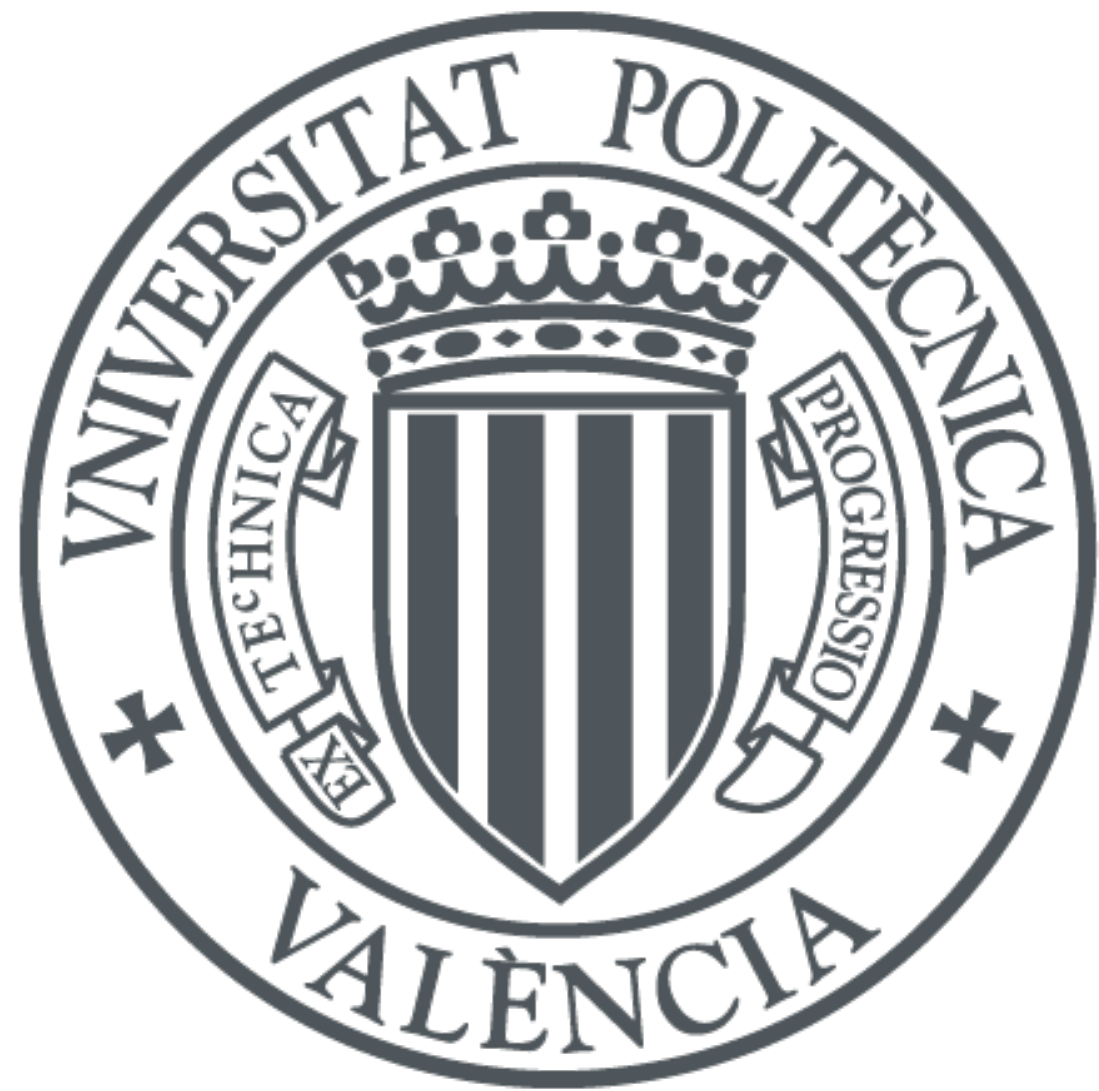

The final publication is available at

http://dx.doi.org/10.1016/j.amc.2015.03.128

Copyright Elsevier

Additional Information 


\title{
A probabilistic estimation and prediction technique for dynamic continuous social science models: The evolution of the attitude of the Basque Country population towards ETA as a case study
}

\author{
Juan-Carlos Cortés $^{\mathrm{a}}$, Francisco-J. Santonja ${ }^{\mathrm{b}}$, Ana-C. Tarazona ${ }^{\mathrm{a}}$, Rafael-J. \\ Villanueva $^{\mathrm{a}, *}$, Javier Villanueva-Oller ${ }^{\mathrm{c}}$ \\ ${ }^{a}$ Instituto Universitario de Matemática Multidisciplinar, Universitat Politècnica de \\ València, Valencia (Spain) \\ ${ }^{b}$ Departamento de Estadística e Investigación Operativa, Universitat de Valéncia, Valencia \\ (Spain) \\ ${ }^{c}$ Centro de Estudios Superiores Felipe II, Aranjuez, Madrid (Spain)
}

\begin{abstract}
In this paper, a computational technique to deal with uncertainty in dynamic continuous models in Social Sciences is presented. Considering data from surveys, the method consists of determining the probability distribution of the survey output and this allows to sample data and fit the model to the sampled data using a goodness-of-fit criterion based on the $\chi^{2}$-test. Taking the fitted parameters that were not rejected by the $\chi^{2}$-test, substituting them into the model and computing their outputs, $95 \%$ confidence intervals in each time instant capturing the uncertainty of the survey data (probabilistic estimation) is built. Using the same set of obtained model parameters, a prediction over the next few years with 95\% confidence intervals (probabilistic prediction) is also provided. This technique is applied to a dynamic social model describing the evolution of the attitude of the Basque Country population towards the revolutionary organization ETA.
\end{abstract}

Keywords: Social dynamic models, Probabilistic estimation, Probabilistic prediction, Attitude dynamics

\section{Introduccion}

Uncertainty quantification in dynamic continuous models is an emerging area [1]. Because of the numerous complex factors that usually involve social behav-

\footnotetext{
${ }^{*}$ Corresponding Author.

Email addresses: jccortes@imm.upv.es (Juan-Carlos Cortés), francisco.santonja@uv.es (Francisco-J. Santonja), actarazona@asic.upv.es (Ana-C. Tarazona), rjvillan@imm.upv.es (Rafael-J. Villanueva), jvillanueva@pdi.ucm.es (Javier Villanueva-Oller)
} 
ior, it is particularly appropriate the consideration of randomness in this kind of models. In practice, the introduction of randomness in continuous models can be done using different approaches. Stochastic differential equations of Itô-type consider uncertainty through a stochastic process called white noise, i.e., the derivative of a Wiener process. As a consequence, this approach limitates the introduction of uncertainty to a Gaussian process whose sample trajectories are somewhat irregular since they are nowhere differentiable. A more convenient approach in social modelling is to permit that input parameters can become random variables and/or stochastic processes and, therefore can follow other type of probability distributions apart from Gaussian. This approach leads to continuous models usually referred to as random differential equations (r.d.e.'s). In dealing with r.d.e.'s, generalized Polynomial Chaos (gPC) is one of the most fruitful methods $[2,3]$.

Most of the existing methods and techniques, assume that input model parameters have known standard probability distributions. In general, setting the probability distribution of the model parameters, standard or empirical, is a crucial and difficult task currently under study which is required for uncertainty modeling approaches.

Also, the computation is an important issue in dealing with uncertainty. For instance, gPC technique may not be affordable when the number of model parameters which are assumed random variables, increases, or the interval where the mean and the standard deviation are valid may be very short [4]. It may turn these techniques inappropriate for modeling real problems.

On the other hand, if we consider that there is not information available for setting the model parameters probabilistic distribution, techniques as bootstrapping [5, 8] or Bayesian [6] are other useful approaches. Related to these statistical techniques, in this paper we propose a computational approach where the data, retrieved from surveys, play a fundamental role to introduce the uncertainty, in estimation and prediction, from the very beginning. This probabilistic approach is applied to a model describing the evolution of the attitude of the Basque population towards the revolutionary organization ETA [7] presented in [8]. In this latter paper, the authors apply the bootstrapping technique to the model in order to deal with uncertainty with the system of differential equations, because we do not assume error in the data. As we will see in the present paper, the uncertainty comes with the data (data sample error) and we will propose new techniques to make the model captures the data uncertainty.

The paper is organized as follows. In Section 2, we summarize the model building described in [8]. In Section 3 we propose a technique which will allow us to obtain a set of model parameters that provide $95 \%$ confidence intervals (95\% CI) for each time instant such that the data uncertainty is captured. We will call this technique probabilistic estimation. With the set of parameters obtained in Section 3, in Section 4 we obtain a probabilistic prediction of the attitude towards ETA of the people of the Basque Country over the next four years. In Section 5, we discuss the results and present the conclusion. 


\section{Model building}

In [8], a deterministic mathematical model was introduced, and series data were retrieved from the Euskobarometro of November 2012 on the attitude of the Basque Country population towards ETA [9, Table 20]. The eight types of attitudes towards ETA that appear in the Euskobarometro (Total support; Justification with criticism; Goals yes / Means no; Before yes / Not now; Indifferent; ETA scares; Total rejection; No answer) were simplified to only three (Support; Rejection; Abstention) and allowed us to divide Basque Country population into the following three subpopulations, time $t$ in years (see [8] for more details):

- Supporters. $A_{1}(t)$ denotes the percentage of people in the Basque Country who have an attitude of support towards ETA at the time instant $t$,

- Rejectors. $A_{2}(t)$ denotes the percentage of people in the Basque Country who have an attitude of rejection towards ETA at the time instant $t$,

- Abstentionists. $A_{3}(t)$ denotes the percentage of people in the Basque Country whose attitude towards ETA is not defined (indifferent), abstain or simply they do not want to declare their opinion, at the time instant $t$.

Data in these three groups appear, in percentages, in Figure 1 from May 1995 until November 2012. In May 2005 the Spanish Parliament approved the possibility the Government to support dialogue with ETA. This fact has been considered as a substantial change in the anti-terrorist policy. This policy is still in force and it justifies that we have chosen this time instant as our model initial condition. In Table 1, the figures in percentages of each subpopulation from May 2005 to November 2012 are presented.
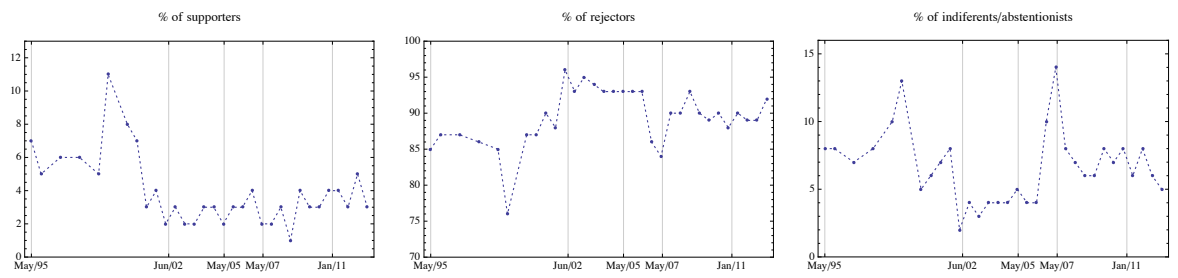

Figure 1: Percentage of Basque Country population with an attitude of support, rejection or abstention towards ETA since May 1995 until November 2012. Vertical lines correspond to remarkable dates: in Jun 2002 the Law of Political Parties was passed and left-wing nationalist political parties were outlawed because their proven relation with ETA; in May 2005 the Spanish Parliament approved the possibility the Government to support dialogue with ETA; in May 2007 left-wing nationalist parties could present candidates again; in Jan 2011 ETA announced a permanent cease-fire. Observe that large jumps in the Rejection population correspond to large jumps in the Abstention population, but in the opposite sense. Supporting population remains with minor variations since the Law of Political Parties passed.

As it was shown in [8], the following system of nonlinear differential equations describes the evolution of attitudes towards ETA in the Basque Country over the time: 


\begin{tabular}{|l|c|c|c|}
\hline Survey date & Support (\%) & Rejection (\%) & Abstention (\%) \\
\hline May 05 & 2 & 93 & 5 \\
Nov. 05 & 3 & 93 & 4 \\
May 06 & 3 & 93 & 4 \\
Nov. 06 & 4 & 86 & 10 \\
May 07 & 2 & 84 & 14 \\
Nov. 07 & 2 & 90 & 8 \\
May 08 & 3 & 90 & 7 \\
Nov. 08 & 1 & 93 & 6 \\
May 09 & 4 & 90 & 6 \\
Nov. 09 & 3 & 89 & 8 \\
May 10 & 3 & 90 & 7 \\
Nov. 10 & 4 & 88 & 8 \\
May 11 & 4 & 90 & 6 \\
Nov. 11 & 3 & 89 & 8 \\
May 12 & 5 & 89 & 6 \\
Nov. 12 & 3 & 92 & 5 \\
\hline
\end{tabular}

Table 1: Percentage of people in the Basque Country with respect to their attitude towards ETA from May 2005 to November 2012.

$$
\begin{aligned}
& A_{1}^{\prime}(t)=\beta_{21} A_{2}(t) A_{1}(t)-\beta_{12} A_{1}(t) A_{2}(t)+\beta_{31} A_{3}(t) A_{1}(t)-\beta_{13} A_{1}(t) A_{3}(t), \\
& A_{2}^{\prime}(t)=\beta_{12} A_{1}(t) A_{2}(t)-\beta_{21} A_{2}(t) A_{1}(t)+\beta_{32} A_{3}(t) A_{2}(t)-\beta_{23} A_{2}(t) A_{3}(t), \\
& A_{3}^{\prime}(t)=\beta_{13} A_{1}(t) A_{3}(t)-\beta_{31} A_{3}(t) A_{1}(t)+\beta_{23} A_{2}(t) A_{3}(t)-\beta_{32} A_{3}(t) A_{2}(t) .
\end{aligned}
$$

Taking $\gamma_{12}=\beta_{12}-\beta_{21}, \gamma_{13}=\beta_{13}-\beta_{31}$ and $\gamma_{23}=\beta_{23}-\beta_{32}$, the above system can be simplified as follows

$$
\begin{aligned}
& A_{1}^{\prime}(t)=-\gamma_{12} A_{2}(t) A_{1}(t)-\gamma_{13} A_{3}(t) A_{1}(t), \\
& A_{2}^{\prime}(t)=\gamma_{12} A_{2}(t) A_{1}(t)-\gamma_{23} A_{3}(t) A_{2}(t), \\
& A_{3}^{\prime}(t)=\gamma_{13} A_{3}(t) A_{1}(t)+\gamma_{23} A_{3}(t) A_{2}(t) .
\end{aligned}
$$

Note that if $\gamma_{i j}>0$ the net movement of individuals is from $A_{i}$ to $A_{j}$. The above system of differential equations can be represented by the diagram of Figure 2. See [8] for more details.

\section{Probabilistic estimation: A computational technique to determine the empirical probabilistic distribution of model parameters}

\subsection{Data}

Data collected in Table 1 correspond to the mean percentage obtained from the Euskobarometro surveys since May 2005 to November 2012 [9, Table 20]. In 


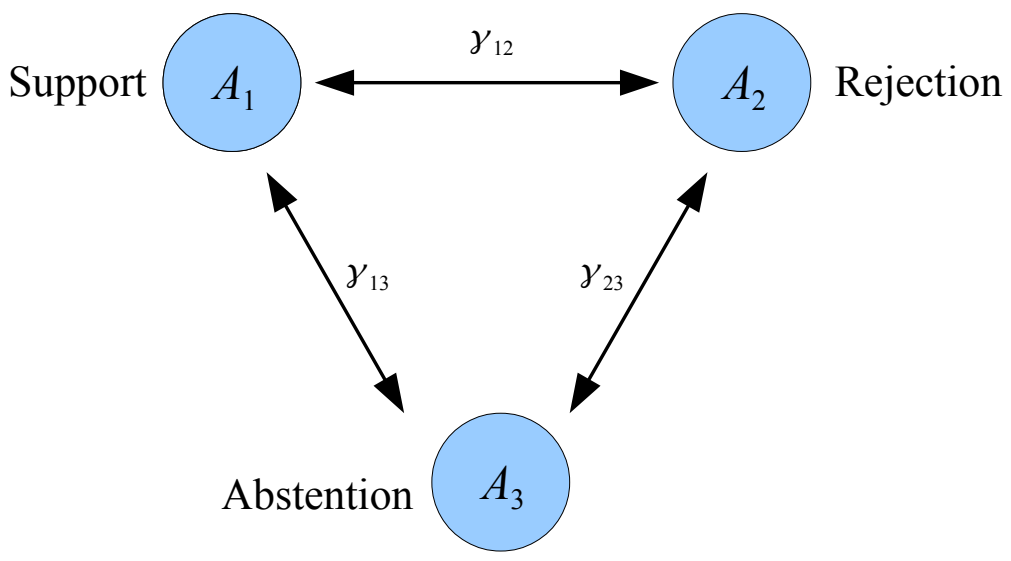

Figure 2: Graph depicting the model (1)-(3). The circles represent the subpopulations and the arrows denote the flow of people who change their attitude towards ETA over the time.

the technical specifications of each survey, 1800 and 1200 interviews are reported (see column 3 "Sample size" in Table 2).

Taking into account that the sample is not the same for each survey, let us assume that the survey outputs are independent. For each one of the 16 available surveys, let us denote by $X^{j}=\left(X_{1}^{j}, X_{2}^{j}, X_{3}^{j}\right), 0 \leq X_{i}^{j} \leq n_{j}, i=1,2,3$, $j=1, \ldots, 16$, a random vector whose entries are $X_{1}^{j}=$ Support, $X_{2}^{j}=$ Rejection, $X_{3}^{j}=$ Abstention and $n_{j} \in\{1200,1800\}$ is the sample size of survey $j$. These components represent exclusive selections (events) with probabilities

$$
P^{j}\left(X_{1}^{j}=x_{1}\right)=\theta_{1}^{j}, P^{j}\left(X_{2}^{j}=x_{2}\right)=\theta_{2}^{j}, P^{j}\left(X_{3}^{j}=x_{3}\right)=\theta_{3}^{j}, \quad j=1, \ldots, 16,
$$

where $\theta_{1}^{j}, \theta_{2}^{j}$ and $\theta_{3}^{j}$ are the percentages collected in Table 1 for each survey $j, j=1, \ldots, 16$. Thus, each random vector $X^{j}$ has a multinomial (trinomial) probability distribution. Therefore, the probability that $X_{1}^{j}$ occurs $x_{1}$ times, $X_{2}^{j}$ occurs $x_{2}$ times and $X_{3}^{j}$ occurs $x_{3}$ times is given by

$$
P_{n_{j}}^{j}\left(x_{1}, x_{2}, x_{3}\right)=\frac{n_{j} !}{x_{1} ! x_{2} ! x_{3} !}\left(\theta_{1}^{j}\right)^{x_{1}}\left(\theta_{2}^{j}\right)^{x_{2}}\left(\theta_{3}^{j}\right)^{x_{3}}, j=1, \ldots, 16,
$$

where $x_{1}+x_{2}+x_{3}=n_{j}$. The resulting trinomials for each Euskobarometro survey can be seen in the last column "Joint trinomial probability function" in Table 2 .

\subsection{Probabilistic estimation}

In this section, we are going to sample data from each survey using the joint trinomial distribution set in Table 2. This will be carried out a high 


\begin{tabular}{|c|c|c|c|}
\hline & $\begin{array}{c}\text { Survey } \\
\text { dates }\end{array}$ & $\begin{array}{l}\text { Sample } \\
\text { size }\end{array}$ & Joint trinomial probability function \\
\hline$j=1$ & $t_{1}=$ May 05 & $n_{1}=1800$ & $P_{1800}^{1}\left(x_{1}, x_{2}, x_{3}\right)=\frac{1800 !}{x_{1} ! x_{2} ! x_{3} !} 0.02^{x_{1}} 0.93^{x_{2}} 0.05^{x_{3}}$ \\
\hline$j=2$ & $t_{2}=$ Nov. 05 & $n_{2}=1200$ & $P_{1200}^{2}\left(x_{1}, x_{2}, x_{3}\right)=\frac{1200 !}{x_{1} ! x_{2} ! x_{3} !} 0.03^{x_{1}} 0.93^{x_{2}} 0.04^{x_{3}}$ \\
\hline$j=3$ & $t_{3}=$ May 06 & $n_{3}=1800$ & $P_{1800}^{3}\left(x_{1}, x_{2}, x_{3}\right)=\frac{1800 !}{x_{1} ! x_{2} ! x_{3} !} 0.03^{x_{1}} 0.93^{x_{2}} 0.04^{x_{3}}$ \\
\hline$j=4$ & $t_{4}=$ Nov. 06 & $n_{4}=1200$ & $P_{1200}^{4}\left(x_{1}, x_{2}, x_{3}\right)=\frac{1200 !^{3}}{x_{1} ! x_{2} ! x_{3} !} 0.04^{x_{1}} 0.86^{x_{2}} 0.1^{x_{3}}$ \\
\hline$j=5$ & $t_{5}=$ May 07 & $n_{5}=1200$ & $P_{1200}^{5}\left(x_{1}, x_{2}, x_{3}\right)=\frac{1200 !}{x_{1} ! x_{2} ! x_{3} !} 0.02^{x_{1}} 0.84^{x_{2}} 0.14^{x_{3}}$ \\
\hline$j=6$ & $t_{6}=$ Nov. 07 & $n_{6}=1200$ & $P_{1200}^{6}\left(x_{1}, x_{2}, x_{3}\right)=\frac{1200 !}{x_{1} ! x_{2} ! x_{3} !} 0.02^{x_{1}} 0.9^{x_{2}} 0.08^{x_{3}}$ \\
\hline$j=7$ & $t_{7}=$ May 08 & $n_{7}=1800$ & $P_{1800}^{7}\left(x_{1}, x_{2}, x_{3}\right)=\frac{1800 !}{x_{1} ! x_{2} ! x_{3} !} 0.03^{x_{1}} 0.9^{x_{2}} 0.07^{x_{3}}$ \\
\hline$j=8$ & $t_{8}=$ Nov. 08 & $n_{8}=1200$ & $P_{1200}^{8}\left(x_{1}, x_{2}, x_{3}\right)=\frac{1200 !}{x_{1} ! x_{2} ! x_{3} !} 0.01^{x_{1}} 0.93^{x_{2}} 0.06^{x_{3}}$ \\
\hline$j=9$ & $t_{9}=$ May 09 & $n_{9}=1200$ & $P_{1200}^{9}\left(x_{1}, x_{2}, x_{3}\right)=\frac{1220 ?^{3}}{x_{1} ! x_{2} ! x_{3} !} 0.04^{x_{1}} 0.9^{x_{2}} 0.06^{x_{3}}$ \\
\hline$j=10$ & $t_{10}=$ Nov. 09 & $n_{10}=1200$ & $P_{1200}^{10}\left(x_{1}, x_{2}, x_{3}\right)=\frac{1200 !}{x_{1} ! x_{2} ! x_{3} !} 0.03^{x_{1}} 0.89^{x_{2}} 0.08^{x_{3}}$ \\
\hline$j=11$ & $t_{11}=$ May 10 & $n_{11}=1200$ & $P_{1200}^{11}\left(x_{1}, x_{2}, x_{3}\right)=\frac{1200 !}{x_{1} ! x_{2} ! x_{3} !} 0.03^{x_{1}} 0.9^{x_{2}} 0.07^{x_{3}}$ \\
\hline$j=12$ & $t_{12}=$ Nov. 10 & $n_{12}=1200$ & $P_{1200}^{12}\left(x_{1}, x_{2}, x_{3}\right)=\frac{1200 !}{x_{1} ! x_{2} ! x_{3} !} 0.04^{x_{1}} 0.88^{x_{2}} 0.08^{x_{3}}$ \\
\hline$j=13$ & $t_{13}=$ May 11 & $n_{13}=1200$ & $P_{1200}^{13}\left(x_{1}, x_{2}, x_{3}\right)=\frac{1200 !}{x_{1} ! x_{2} ! x_{3} !} 0.04^{x_{1}} 0.9^{x_{2}} 0.06^{x_{3}}$ \\
\hline$j=14$ & $t_{14}=$ Nov. 11 & $n_{14}=1200$ & $P_{1200}^{14}\left(x_{1}, x_{2}, x_{3}\right)=\frac{1200 !}{x_{1} ! x_{2} ! x_{3} !} 0.03^{x_{1}} 0.89^{x_{2}} 0.08^{x_{3}}$ \\
\hline$j=15$ & $t_{15}=$ May 12 & $n_{15}=1200$ & $P_{1200}^{15}\left(x_{1}, x_{2}, x_{3}\right)=\frac{1200 !}{x_{1} ! x_{2} ! x_{3} !} 0.05^{x_{1}} 0.89^{x_{2}} 0.06^{x_{3}}$ \\
\hline$j=16$ & $t_{16}=$ Nov. 12 & $n_{16}=1200$ & $P_{1200}^{16}\left(x_{1}, x_{2}, x_{3}\right)=\frac{1200 !}{x_{1} ! x_{2} ! x_{3} !} 0.03^{x_{1}} 0.92^{x_{2}} 0.05^{x_{3}}$ \\
\hline
\end{tabular}

Table 2: Data for probabilistic model estimation. Date, sample size and joint trinomial probability function of each survey.

number of times ( $10^{4}$ times) in order to generate a representative sample for each survey. Every time we sample data survey, we determine the model parameter estimations $\gamma_{12}, \gamma_{13}, \gamma_{23}$, using the Nelder-Mead optimization algorithm [11, 12] with goodness-of-fit $\chi^{2}$-test [10]. The parameters with $p$-value less than 0.05 will be rejected. The remainder will be sorted by $p$-value descending order. Selecting some of these model parameter vectors, we will be able to use the model outputs to provide a confidence band determined by the percentiles 2.5 and 97.5 (95\% Confidence Interval (CI)) in each time instant. This 95\% Model Confidence Band (95\% MCB) is what we define as probabilistic estimation. Let us describe in detail the procedure.

1. Compute the quantiles 2.5 and 97.5 (95\% CI) of each one of the joint multinomial distributions in Table $2, j=1,2, \ldots, 16$, for Support, Rejection and Abstention subpopulations sampling multinomials a hundred thousand times, obtaining

$$
\begin{aligned}
Q_{2.5}^{\text {support }}= & (1.39,2.08,2.22,2.92,1.25,1.25,2.22,0.50,2.92, \\
& 2.08,2.08,2.92,2.92,2.08,3.83,2.08), \\
Q_{97.5}^{\text {support }}= & (2.67,4.00,3.78,5.17,2.83,2.83,3.83,1.58,5.17, \\
& 4.00,4.00,5.17,5.17,4.00,6.25,4.00), \\
Q_{2.5}^{\text {reject }}= & (91.80,91.50,91.80,84.00,81.90,88.20,88.60,91.50, \\
& 88.20,87.20,88.20,86.20,88.20,87.20,87.20,90.40),
\end{aligned}
$$




$$
\begin{aligned}
Q_{97.5}^{\text {reject }}= & (94.20,94.40,94.20,87.90,86.10,91.70,91.40,94.40, \\
& 91.70,90.70,91.70,89.80,91.70,90.70,90.70,93.50), \\
Q_{2.5}^{\text {abstention }}= & (4.00,2.92,3.11,8.33,12.10,6.50,5.83,4.67,4.67, \\
& 6.50,5.58,6.50,4.67,6.50,4.67,3.83), \\
Q_{97.5}^{\text {abstention }}= & (6.00,5.17,4.94,11.80,16.00,9.58,8.22,7.33,7.33, \\
& 9.58,8.50,9.58,7.33,9.58,7.42,6.25) .
\end{aligned}
$$

The $95 \%$ CI determined by the above percentiles constitute an approximation of the survey results. These 95\% CI have been drawn in Figures 3 and 4 as vertical segments (error bars). Moreover, these $95 \%$ CI will be used to find the best probabilistic estimation.

2. Let us define the following function of the parameters $\gamma_{12}, \gamma_{13}$ and $\gamma_{23}$ :

(A) For given values of $\gamma_{12}, \gamma_{13}$ and $\gamma_{23}$ parameters, compute the model output in $t_{1}=$ May 2005, $t_{2}=$ November $2005, \ldots, t_{15}=$ May 2012 and $t_{16}=$ November 2012 for the three subpopulations, Support, Rejection and Abstention.

(B) For each subpopulation, compare the model output obtained in step (2A) with the data values we will sample in step (3A) using the $\chi^{2}$-test and then, obtain a $p$-value for each subpopulation.

(C) Calculate the minimum $p$-value among the three previous $p$-values.

3. For $i=1$ to $10^{4}$

(A) Sample another set of values of all the trinomial distributions collected in Table 2. Then, we will have one sample of 16 surveys with percentages for Support, Rejection and Abstention populations from May 2005 until November 2012. Therefore, we will have a set of sampled data as in Table 1.

(B) Find the model parameter values $\gamma_{12}^{i}, \gamma_{13}^{i}$ and $\gamma_{23}^{i}$ with the highest $p$-value (maximizing the function defined in steps $(2 \mathrm{~A}),(2 \mathrm{~B})$ and $(2 \mathrm{C})$ ). To do that, Nelder-Mead optmization algorithm is used [11, 12] using as a goodness-of-fit the $\chi^{2}$-test.

4. Once the above process is completed, store the obtained parameter values and the $p$-value as the vector

$$
\left(\gamma_{12}^{i}, \gamma_{13}^{i}, \gamma_{23}^{i}, p-\text { value }_{i}\right), 1 \leq i \leq 10^{4} .
$$

5. Reject the model parameters with $p$-value less than 0.05 . In our case, 4990 out of $10^{4}$ satisfy this restriction. Then, they are sorted by $p$-value descending order as follows,

$$
\left(\gamma_{12}^{i}, \gamma_{13}^{i}, \gamma_{23}^{i}, p-\text { value }_{i}\right), 1 \leq i \leq 4990 .
$$

6. For $k=2$ to 4990 
(A) Substitute into the model the parameters $\left(\gamma_{12}^{j}, \gamma_{13}^{j}, \gamma_{23}^{j}\right)$, for $j=$ $1,2, \ldots, k$, and compute the model output in $t_{1}=$ May $2005, t_{2}=$ November 2005, .., $t_{15}=$ May 2012 and $t_{16}=$ November 2012.

a1) Take the $k$ model outputs for Support, Rejection and Abstention at the time instant $t_{1}=$ May 2005 and calculate the corresponding quantiles 2.5 and 97.5 (95\% CI).

a2) Take the $k$ model outputs for Support, Rejection and Abstention at the time instant $t_{2}=$ November 2005 and calculate the corresponding quantiles 2.5 and $97.5(95 \% \mathrm{CI})$.

$-\cdots$

a16) Take the $k$ model outputs for Support, Rejection and Abstention at the time instant $t_{16}=$ November 2012 and calculate the corresponding quantiles 2.5 and 97.5 (95\% CI).

(B) Now, gather the calculated quantiles 2.5 for Support, Rejection and Abstention subpopulations and store them sequentially on the vectors $S_{2.5}^{k}, R_{2.5}^{k}$ and $A_{2.5}^{k}$, respectively.

(C) Gather the calculated quantiles 97.5 for Support, Rejection and Abstention subpopulations and store them sequentially on the vectors $S_{97.5}^{k}, R_{97.5}^{k}$ and $A_{97.5}^{k}$, respectively.

(D) Calculate the $p$-values using the $\chi^{2}$-test to datasets obtained in steps $(1),(6 \mathrm{~B})$ and $(6 \mathrm{C})$ grouped in pairs as follows,

d1) $Q_{2.5}^{\text {support }}$ and $S_{2.5}^{k}$,

d2) $Q_{97.5}^{\text {support }}$ and $S_{97.5}^{k}$,

d3) $Q_{2.5}^{\text {reject }}$ and $R_{2.5}^{k}$,

d4) $Q_{97.5}^{\text {reject }}$ and $R_{97.5}^{k}$,

d5) $Q_{2.5}^{\text {abstention }}$ and $A_{2.5}^{k}$,

d6) $Q_{97.5}^{a b s t e n t i o n}$ and $A_{97.5}^{k}$.

Note that, in order to know the parameter values which allow us to define the $95 \%$ MCB (probabilistic estimation), we compare percentil vectors obtained by the trinomial sampling with the ones obtained using the model outputs considering the 4990 optimal values.

E) Calculate $m_{k}$, the minimum $p$-value among the six previous $p$-values and build the pair $\left(k, m_{k}\right)$.

7. Select the pair $\left(k, m_{k}\right)$ among the $4990-1$ with the maximum $m_{k}$.

In our case, the obtained value is $k=77$ with $m_{77}=0.972991$ and, consequently, the $p$-values corresponding to percentiles 2.5 and 97.5 for each subpopulation are greater than $m_{77}$.

Now, we take the $k=77$ set of parameters obtained in the above procedure, compute the model output from $t_{1}=$ May 2005 to $t_{16}=$ November 2012, in jumps of 0.05 and, in each point, we calculate the percentiles 2.5 and 97.5 

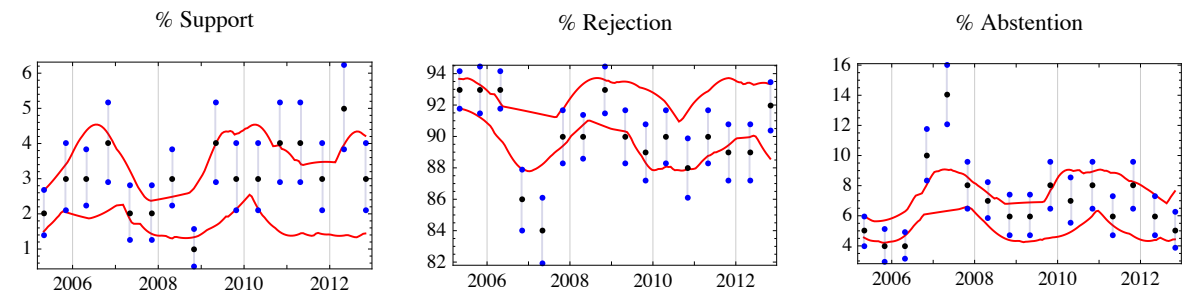

Figure 3: Probabilistic estimation. The vertical segments (error bars) correspond to the 95\% CI of the simulated data using trinomial distributions appearing in Table 2. The points in the middle of the segments are the mean values in Table 1. The continuous lines are the model 95\% MCB (probabilistic estimation) obtained with the described procedure. Note that most of the segments cross continuous lines determined by the model, capturing the data uncertainty. Only for Rejection and Abstention subpopulations in time instants November 2006 and May 2007 the uncertainty is not captured.

for each subpopulation (95\% MCB). The result (probabilistic estimation) is depicted in Figure 3 as red continuous lines.

The vertical segments (error bars) correspond to the $95 \%$ CI of the survey data simulated by trinomial distributions appearing in Table 2. The points in the middle of the segments are the mean values collected in Table 1 . The continuous lines are the model $95 \% \mathrm{MCB}$ obtained from the model outputs of the first $k=77$ out of 4990 sets of model parameters that best fit the samples of the trinomial distributions in Table 2.

\subsection{Probabilistic estimation analysis}

The idea of the probabilistic estimation described in the previous section is to obtain $95 \%$ model confidence interval bands (MCB) as close as possible, in the $\chi^{2}$-test sense, to $95 \%$ CI of the data distributions appearing in Table 2 (vertical segments in Figure 3). This closeness depends on both the model and the data. In general, in Social Sciences and, in particular in our case, these data are very sensitive to punctual events, not considered explicitly through model hypotheses, that in the model under study, may affect the attitude towards ETA.

It is remarkable to note that the application of $\chi^{2}$-test in the procedure of the previous section to select the best fittings, allowed us to find 4990 sets of model parameters for which the model estimation cannot be rejected as explanation of the data representing the phenomenon under study. In fact, we also could select the best (highest $p$-value) among all of them.

In addition, looking at the graphics in Figure 3, we can see that almost all the vertical segments (error bars) cross at least a continuous line indicating that data uncertainty is captured by the model, in particular for Support subpopulation.

An especial mention deserves the Rejection and Abstention subpopulation graphics, where we can distinguish two parts. The first one, from May 2005 to May 2007, the probabilistic estimation intends to follow the data trajectory 
but the data uncertainty in November 2006 and May 2007 is not captured when sudden jumps happen. As we mentioned in Figure 1, large jumps in the Rejection population correspond to large jumps in the Abstention population, in the opposite sense. We consider that the jumps in November 2006 and May 2007 are due to certain events that occurred from Sep 2006 to May 2007 as: increasing of vandalism acts from Sep 2006 to Dec 2006 linked with young leftwing nationalist groups; Barajas Airport Terminal 4 attack claimed by ETA (Dec 2006); in May 2007 local elections, the left-wing nationalist party EAEANV was allowed to present candidates in some villages and cities. In the second part, from November 2007 until November 2012, the continuous lines capture the data uncertainty.

Therefore, even though the estimation for Rejection and Abstention subpopulations do not capture the data uncertainty in two time instants, the three subpopulations capture the remainder. This leads us to consider the model and its probabilistic estimation appropriate to provide a prediction of the evolution of the population's attitude towards ETA over the next four years.

\section{Probabilistic predictions over the next four years}

Now, taking the model and the $k=77$ set of parameters obtained in the probabilistic estimation, we are going to give the probabilistic prediction over the next four years by computing the model outputs from November 2012 to November 2016 and then, obtaining the 95\% MCB (model continuous lines). We show the results graphically in Figure 4 and some numerical values in Table 3 .
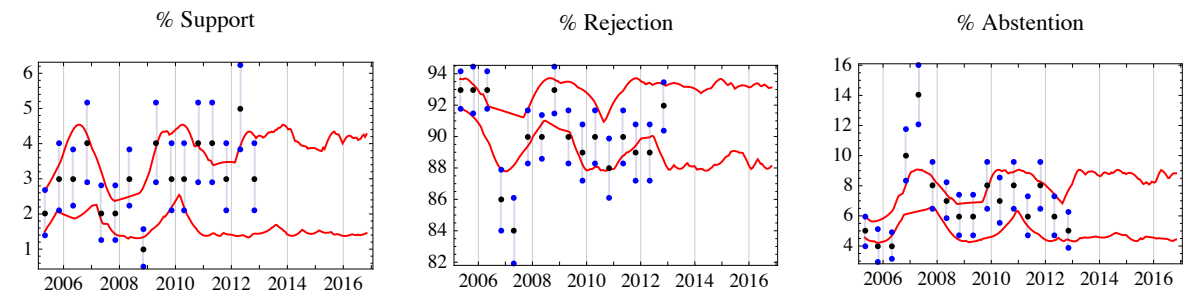

Figure 4: Probabilistic prediction. These graphs have been plotted from the Figure 3 including the predictions over the next four years as $95 \%$ MCB (model continuous lines).

Figure 4 and Table 3 show that the attitude towards ETA of the population living in the Basque Country will remain fairly stable over the next four years.

\subsection{Robustness of the proposed method}

Note that if we run the proposed procedure again, taking into account that the multinomial sampling is random, we may obtain a different value of $k$, however, we will see that the corresponding $m_{k}$ will be very similar. In fact, we did it twice more obtaining $k=129$ and $k=84$ with $m_{k}=0.9624$ and 


\begin{tabular}{|c|c|c|c|c|c|c|}
\hline Date & \multicolumn{2}{|c|}{ Support } & \multicolumn{2}{c|}{ Rejection } & \multicolumn{2}{c|}{ Abstention } \\
& Mean & 95\% CI & Mean & 95\% CI & Mean & $95 \%$ CI \\
\hline May 2013 & 3.10 & {$[1.54,4.32]$} & 90.69 & {$[88.02,93.38]$} & 6.22 & {$[4.52,9.01]$} \\
Nov. 2013 & 2.98 & {$[1.55,4.54]$} & 90.28 & {$[88.11,93.15]$} & 6.74 & {$[4.63,8.87]$} \\
May 2014 & 2.68 & {$[1.41,4.06]$} & 90.40 & {$[87.87,92.90]$} & 6.92 & {$[4.63,8.78]$} \\
Nov. 2014 & 2.43 & {$[1.43,3.88]$} & 90.86 & {$[88.21,93.55]$} & 6.71 & {$[4.74,8.90]$} \\
May 2015 & 2.44 & {$[1.47,3.84]$} & 91.23 & {$[89.03,93.41]$} & 6.34 & {$[4.33,8.66]$} \\
Nov. 2015 & 2.62 & {$[1.42,4.26]$} & 91.22 & {$[88.76,93.25]$} & 6.17 & {$[4.60,8.08]$} \\
May 2016 & 2.72 & {$[1.42,4.17]$} & 91.01 & {$[88.56,93.39]$} & 6.27 & {$[4.49,8.99]$} \\
Nov. 2016 & 2.72 & {$[1.46,4.28]$} & 90.87 & {$[88.13,93.13]$} & 6.41 & {$[4.43,8.82]$} \\
\hline
\end{tabular}

Table 3: Mean and 95\% confidence interval predictions for the coming eight Euskobarometro surveys.

$m_{k}=0.966348$, respectively. The probabilistic predictions in these two new cases are given in Tables 4 and 5 . We can see that the predictions were very similar. This shows the robustness of the proposed method.

\begin{tabular}{|c|c|c|c|c|c|c|}
\hline Date & \multicolumn{2}{|c|}{ Support } & \multicolumn{2}{c|}{ Rejection } & \multicolumn{2}{c|}{ Abstention } \\
& Mean & $95 \%$ CI & Mean & 95\% CI & Mean & 95\% CI \\
\hline May 2013 & 3.08 & {$[1.48,4.76]$} & 91.02 & {$[88.40,93.44]$} & 5.90 & {$[4.42,8.33]$} \\
Nov. 2013 & 3.09 & {$[1.59,4.61]$} & 90.41 & {$[87.90,93.33]$} & 6.50 & {$[4.35,8.97]$} \\
May 2014 & 2.86 & {$[1.39,4.52]$} & 90.30 & {$[88.05,92.89]$} & 6.84 & {$[4.43,8.89]$} \\
Nov. 2014 & 2.59 & {$[1.48,4.22]$} & 90.64 & {$[88.50,93.28]$} & 6.77 & {$[4.15,8.71]$} \\
May 2015 & 2.45 & {$[1.46,3.86]$} & 91.10 & {$[88.54,93.21]$} & 6.45 & {$[3.89,8.53]$} \\
Nov. 2015 & 2.51 & {$[1.56,3.93]$} & 91.35 & {$[88.98,93.31]$} & 6.14 & {$[3.67,8.41]$} \\
May 2016 & 2.67 & {$[1.47,4.32]$} & 91.28 & {$[88.79,93.33]$} & 6.05 & {$[3.56,8.29]$} \\
Nov. 2016 & 2.75 & {$[1.31,4.40]$} & 91.06 & {$[88.09,93.19]$} & 6.19 & {$[3.74,8.75]$} \\
\hline
\end{tabular}

Table 4: Mean and 95\% confidence interval predictions for the coming eight Euskobarometro surveys for the second procedure execution, $k=129$ and $m_{k}=0.9624$.

Also, we should say that last June 27th, 2013 was published the Euskobarometro of May 2013 with 1200 interviews. Its figures are shown in Table 6 . The $95 \%$ confidence intervals to this last Euskobarometro were calculated as the Step 1 of the procedure described in Section 3.2.

Comparing data in Table 6 with the results collected in Tables 3, 4 and 5, we can see that the data uncertainty in Euskobarometro May 2013 is captured by our predictions in the three tables.

\section{Conclusion}

In this paper, a computational technique to deal with uncertainty (in parameter estimation and output predictions) in dynamic social models based on systems of differential equations is presented. This technique takes data from surveys to introduce the uncertainty into the model from the very beginning and returns $95 \%$ model confidence interval bands that capture the data uncertainty and predict what will happen over the next future. 


\begin{tabular}{|c|c|c|c|c|c|c|}
\hline Date & \multicolumn{2}{|c|}{ Support } & \multicolumn{2}{c|}{ Rejection } & \multicolumn{2}{c|}{ Abstention } \\
& Mean & $95 \%$ CI & Mean & 95\% CI & Mean & $95 \%$ CI \\
\hline May 2013 & 3.13 & {$[1.73,4.79]$} & 90.89 & {$[88.29,93.22]$} & 5.98 & {$[4.47,8.32]$} \\
Nov. 2013 & 3.08 & {$[1.79,4.59]$} & 90.34 & {$[87.87,92.99]$} & 6.57 & {$[4.38,8.81]$} \\
May 2014 & 2.85 & {$[1.47,4.61]$} & 90.31 & {$[88.17,92.28]$} & 6.84 & {$[4.79,8.89]$} \\
Nov. 2014 & 2.56 & {$[1.47,4.23]$} & 90.66 & {$[88.11,92.88]$} & 6.78 & {$[4.79,8.67]$} \\
May 2015 & 2.39 & {$[1.50,3.66]$} & 91.12 & {$[88.65,93.34]$} & 6.50 & {$[4.27,8.51]$} \\
Nov. 2015 & 2.48 & {$[1.54,3.84]$} & 91.34 & {$[89.30,93.05]$} & 6.18 & {$[4.40,8.37]$} \\
May 2016 & 2.67 & {$[1.47,4.26]$} & 91.17 & {$[88.63,93.08]$} & 6.15 & {$[4.71,8.09]$} \\
Nov. 2016 & 2.71 & {$[1.43,4.35]$} & 90.94 & {$[87.93,93.28]$} & 6.35 & {$[4.18,8.69]$} \\
\hline
\end{tabular}

Table 5: Mean and 95\% confidence interval predictions for the coming eight Euskobarometro surveys for the third procedure execution, $k=84$ and $m_{k}=0.966348$.

\begin{tabular}{|c|c|c|c|c|c|c|}
\hline Date & \multicolumn{2}{|c|}{ Support } & \multicolumn{2}{c|}{ Rejection } & \multicolumn{2}{c|}{ Abstention } \\
& Mean & $95 \%$ CI & Mean & $95 \%$ CI & Mean & $95 \%$ CI \\
\hline May 2013 & 3 & {$[2.08,4.00]$} & 89 & {$[87.17,90.75]$} & 8 & {$[6.50,9.58]$} \\
\hline
\end{tabular}

Table 6: Mean and 95\% confidence interval of the Euskobarometro corresponding to May 2013.

The proposed technique has been applied to a mathematical model to study the evolution dynamics of the attitude of Basque population towards ETA in order to illustrate its possibilities. Once the model is stated, we determine a probabilistic estimation in order to find out if the model captures the Euskobarometro data evolution. We observe that the model captures the data uncertainty only partially from May 2005 to May 2007, but from November 2007 the probabilistic estimation improves perceptibly. Anyway, the model estimation is non-rejectable using the $\chi^{2}$-test. Then, we provide a probabilistic prediction of the attitude towards ETA of the population of the Basque Country over the next four years. The predicted results point out that, if the political scenario does not change, the current situation will remain fairly stable.

Additionally, some benefits that can be obtained with this approach are:

- If we consider the model parameters as random variables, the technique presented here as probabilistic estimation allows the estimation of samples of these model parameters (Steps 3, 4 and 5 of the procedure described in Section 3.2). This fact is of paramount interest because one of the main challenges in modeling real problems using random differential equations is to determine the distribution function of model parameters. Therefore, if we use the probabilistic estimation to obtain some samples of the model parameters, in our case $k=77$ parameter samples, we can use these samples together statistical hypothesis testing or kernel functions in order to find distribution functions of the model parameters.

- Other aspect that should be mentioned and it could be interesting for survey prediction estimations is the fact that Table 3 (4 and 5) may be considered as an estimation of the results of the coming Euskobarometro 
surveys (mean and $95 \%$ confidence interval). This idea may be applied to this and other type of surveys where a reliable underlying dynamic model can be built. As a consequence, some surveys may not be carried out with the corresponding saving of money. Therefore, we consider that this approach may be an interesting tool for social behavior studies.

\section{Acknowledgements}

This work has been partially supported by the Spanish M.C.Y.T. grant MTM2013-41765-P.

\section{References}

[1] O.P. Le Maitre, O.M. Knio, Spectral Methods for Uncertainty Quantification, Series Scientific Computation, Springer, Netherlands, 2010.

[2] D. Xiu, Numerical Methods for Stochastic Computations: A Spectral Method Approach, Princeton University Press, New Jersey, 2010.

[3] R. Ghanem, P. Spanos, Stochastic Finite Elements: A Spectral Approach, Dover Publications, New York, 2003.

[4] B.M. Chen-Charpentier, J.-C. Cortes, J.-V. Romero, M.-D. Rosello, Some recommendations for applying gPC (generalized polynomial chaos) to modeling: An analysis through the Airy random differential equation, Appl. Math. Comput. 219 (2013), 4208-4218, http://dx.doi.org/10.1016/j. amc.2012.11.007.

[5] J.C. Cortes, M. Ehrhardt, A. Sanchez-Sanchez, F.J. Santonja, R.J. Villanueva, Modelling the dynamics of the students academic performance in the German region of North Rhine-Westphalia: an epidemiological approach with uncertainty, International Journal of Computer Mathematics, 2014 Vol. 91, No. 2, 241-251, http://dx.doi.org/10.1080/00207160. 2013.813937.

[6] F.C. Coelho, C.T. Codeço, M.G.M. Gomes, A Bayesian framework for parameter estimation in dynamical models, PLoS ONE 6(5):e19616 (2011), http://dx.doi.org/10.1371/journal. pone.0019616.

[7] Communiqué of ETA January 8th 2011. Video published in GARA. Available at http://www.gara.net/bideoak/110108_video/ (in Spanish).

[8] M. Peco, F.-J. Santonja, A.-C. Tarazona, R.-J. Villanueva, J. VillanuevaOller, Effect of the Spanish Law of Political Parties (LPP) on the attitude of the Basque Country population towards ETA: A dynamic modelling approach, Math. Comput. Modell. 57 (2013), 1679-1685, http://dx.doi . org/10.1016/j.mcm.2011.11.007. 
[9] Euskobarometro data series. Available at http://www.ehu.es/ euskobarometro/.

[10] M.H. DeGroot, Probability and Statistics, Addison-Wesley, New York, 1986.

[11] J. A. Nelder, R. Mead, A simplex method for function minimization, Computer Journal 7 (1964), 308-313, http://dx.doi.org/10.1093/comjnl/ 7.4.308.

[12] W.H. Press, B.P. Flannery, S.A. Teukolsky, W. Vetterling, Numerical Recipes: The Art of Scientific Computing, Cambridge University Press, New York, 1986. 